\title{
Outcome Measures in Psoriatic Disease
}

\author{
Lihi Eder • Dafna D. Gladman
}

Published online: 29 June 2013

(C) Springer Science+Business Media New York 2013

\begin{abstract}
Psoriatic arthritis can manifest in various ways, which complicates its assessment. The availability of novel effective therapies required the development of new outcome measurement tools for assessment of the different aspects of the disease in clinical trials and observational studies. Significant progress has been made in our ability to quantify the activity of the different presentations of the disease. A core set of domains was recommended by Outcome Measures in Rheumatology (OMERACT) and Group for Research and Assessment of Psoriasis and Psoriatic Arthritis (GRAPPA) for use in clinical trials and observational studies in patients with psoriatic disease. The core set includes the assessment of skin, nails, joints, enthesitis, dactylitis and spinal disease in addition to patient reported outcomes and quality of life. This manuscript reviews the various measurement tools that are commonly used for the assessment of these domains in patients with psoriatic disease.
\end{abstract}

Keywords Psoriatic disease $\cdot$ Spondyloarthritis · Outcome · Measurement tools · Quality of life

\section{Introduction}

Psoriasis is an immune-mediated skin disease affecting 1-3\% of the population $[1,2]$. Psoriatic arthritis (PsA) is an inflammatory arthritis associated with psoriasis that affects $14 \%$ to $30 \%$ of the patients with psoriasis [3, 4]. Psoriatic arthritis is classified among the seronegative spondyloarthritis ( $\mathrm{SpA}$ ) group, of which the prototype disease is ankylosing spondylitis (AS). Psoriatic arthritis is a multifaceted disease as it manifests in various presentations. Peripheral arthritis is the predominant

L. Eder • D. D. Gladman $(\bowtie)$

Centre for Prognosis Studies in the Rheumatic Diseases,

Toronto Western Hospital, Toronto, ON, Canada

e-mail: dafna.gladman@utoronto.ca manifestation of the disease; however, the pattern of arthritis is different from the one seen in rheumatoid arthritis (RA). The pattern of arthritis in PsA tends to be asymmetric, occasionally affecting only a few joints (oligoarticular arthritis), and tends to involve the lower extremities and the distal interphalangeal (DIP) joints. Furthermore, in approximately half of the patients, the spine is also involved in the form of sacroiliitis or spondylitis [5•]. Another common clinical feature of PsA is enthesitis, an inflammation at the attachment site of tendon or ligament insertion to the bone. Dactylitis, another common feature of PsA, is characterized by diffuse swelling of the entire finger or toe; it affects about half of the patients and is associated with radiographic joint damage [6].

The need for reliable and valid outcome measures for the assessment of disease activity in psoriasis and PsA emerged in the last decade. The development of several novel therapies necessitated their assessment in clinical trials. Furthermore, in rheumatology, the prevention of joint damage by suppression of joint inflammation is now a realistic target with new biologic therapies. The concept of "treat to target", which means that the treatment of arthritis is aimed at achieving minimal disease activity state or remission, requires quantification of disease activity using validated measurement tools [7].

Over the past decade, significant progress has been achieved in the development and validation of new measurement tools for disease activity in psoriatic disease. A core set of domains was recommended by the Outcome Measures in Rheumatology (OMERACT) and Group for Research and Assessment of Psoriasis and Psoriatic Arthritis (GRAPPA) for use in clinical trials and observational studies in patients with psoriatic disease [8]. The six core domains are: peripheral joint activity, skin activity, pain, patient global assessment, physical function, and health-related quality of life. The following domains were considered important but not mandatory: enthesitis, dactylitis, fatigue, nail disease, spinal disease, acute-phase reactant, physician global assessment and radiographic damage to the joints. 
In this article, measurement tools for the above-mentioned domains will be reviewed.

\section{Assessment of the Skin}

No direct link was found between the activity of the skin disease and the joint disease in psoriatic disease [9]. Plaque psoriasis is the most common type of psoriasis, and the one most investigated. Other types of psoriasis include: guttate, pustular and erythroderma [10]. The measurement of severity of plaque psoriasis is based on the level of erythema, infiltration and scaling of the plaque and the extent of body surface area involved. More than 40 different measurement tools have been used in studies and clinical trials for the assessment of psoriasis activity; among them, the Psoriasis Area and Severity Index (PASI), Body Surface Area (BSA) and the Physician Global Assessment (PGA) are the most commonly used tools in clinical trials. PASI combines the assessment of the severity of the lesions and the area affected into a single score. The severity of the skin lesions is scored using four items (erythema, induration, scaling and surface area) for each of the four body areas (head, trunk, upper and lower extremities). The summation of the scores from each of the body areas provides a total PASI score that can range from 0 (no disease) to 72 (most severe) [11]. PASI is the most extensively validated and the most complete score, as it incorporates several items that reflect disease activity. It was found to be reliable compared to objective measures of psoriasis activity, and is highly reproducible [12-14]. It can be reliably performed by dermatologists and clinicians from other specialties after short training $[15,16]$. The selfadministered version of PASI (SAPSI) that is performed by psoriasis patients correlates well with PASI [17]. One of the important qualities of PASI is its responsiveness to change, which is very important in clinical drug trials $[17,18]$. A recently published systematic review that compared several clinical tools for assessment of psoriasis severity concluded that PASI was the most valid and reproducible clinical severity score in the management of patients with plaque psoriasis [19•]. However, PASI has several limitations; one of them is the lack of sensitivity to change at the low end of its range [14]. Therefore, despite its common use in clinical trials, PASI usually serves only as a secondary outcome, and it is not calculated in patients with mild skin involvement (body surface area of less than $3 \%$ ). Furthermore, PASI is based on objective parameters of the skin lesions and does not assess subjective qualities such as pruritus that might be important for the patient. Lastly, nearly half of the scale is redundant, as it is rare to encounter patients with PASI of more than 40. In clinical trials an improvement of at least $75 \%$ in the PASI score (PASI75) is considered to be the standard effective response to the medication, although with the introduction of more effective medications PASI90 response has also been used. Since a significant proportion of the patients with PsA who participate in clinical trials have only mild skin involvement, the assessment of a target lesion is used to measure the activity of psoriasis and its response to the treatment. A measurable target lesion is defined as a lesion of at least $2 \mathrm{~cm}$ in diameter that is located in a body site that available for assessment (not in the scalp or the groin area). The target lesion is evaluated over time for size (in diameter) and erythema, induration and scaling on a scale of 0 to 3 .

Another measurement tool for psoriasis severity is the body surface area (BSA), which is an estimate of the percentage of body surface area affected by psoriasis [20]. The BSA is calculated by the number of patient's hands covering the affected area. The area of a patient's hand represents $1 \%$ of the total BSA. The method is easy and fast to perform; however, it does not consider the severity of plaques. In addition, studies suggested that one hand actually represents $0.70-0.76 \%$ of the BSA, leading to overestimation of BSA, and may also explain the high inter-observer variability [21, 22].

Physician global assessment is another commonly used method to assess psoriasis severity [12,13]. This is a global assessment of the patient's skin lesions on a scale of 0 to 6 , where 0 equals clear skin and 6 is very severe psoriasis. The PGA depends on the investigator's memory of baseline severity of the lesions. Another version of this tool that does not require consideration of the baseline characteristics is called physician's static PGA. Although this method is more subjective, it more closely resembles the assessment made by the dermatologist in clinical practice.

In an international reliability study that included rheumatologists and dermatologists with extensive experience in the assessment of psoriasis and PsA, a substantial agreement on PASI score (ICC 0.74 among dermatologists and 0.70 among rheumatologists), moderate to substantial agreement on PGA (ICC 0.66 among dermatologists and 0.49 among rheumatologists) and substantial agreement on BSA among dermatologists (ICC 0.65), but only fair agreement among rheumatologists (ICC 0.3), was found [15].

\section{Assessment of Nails}

Psoriatic nail lesions affect approximately $40 \%$ of the patients with psoriasis and up to $83 \%$ of the patients with PsA [23]. Their presence is considered a clinical marker for an increased risk of PsA among patients with psoriasis [24]. The nail lesions are divided into lesions that affect the nail bed and those that affect the nail matrix that result in changes in the nail plate. Nail matrix lesions include pitting, leuconychia, red spots in the lunula and nail crumbling, while lesions in the nail bed include onycholysis, subungal hyperkeratosis, oil drop changes and splinter hemorrhage. 
The Nail Psoriasis Severity Index (NAPSI) was the first method developed to assess the severity of nail psoriasis, and has been used in clinical trials to assess response to medication [25]. In this system, the nail is divided into four quadrants. Each quadrant is scored for the presence of matrix and nail bed lesions (a total of $0-4$ for each group for the entire nail and a total nail score of $0-8$ ). The score ranges from 0 to 80 for the fingernails and 0 to 160 if the toenails are also included. The same method is occasionally applied to a target nail, which simplifies the assessment. The NAPSI has been found to be reproducible and responsive to change, and it is routinely used in clinical trials among psoriasis patients $[26,27]$. However, the NAPSI is time-consuming and it is not practical for use in clinical practice. A modification to this method, the modified NAPSI, was developed by rheumatologists and dermatologists for PsA patients, and includes the assessment of each of the above-mentioned features for each nail [28]. Three types of lesions, nail crumbling, pitting and onycholysis, are graded for severity and the remaining lesions are only recorded for their presence in each nail. The method is highly reproducible and correlates well with other clinical features of PsA activity, such as joint count. [28].

\section{Joint Assessment}

The predominant clinical manifestation of PsA is inflammation of the peripheral joints that is characterized by tenderness and/or swelling on palpation at the joint line. The pattern of arthritis tends to be asymmetric and to involve joint areas that are not usually affected in RA, such as the lower extremities and the DIP joints [29, 30]. The assessment of joints for tenderness and swelling is performed by palpation and application of pressure on the joint line. The number of joints in which tenderness or swelling is identified is recorded [31]. In PsA, the standard joint count used in clinical trials includes 66 joints for swelling and 68 joints for tenderness and involves the following joints: DIP, proximal interphalangeal (PIP), metacarpophalangeal (MCP) of the hand, wrist, elbow, shoulder, acromioclavicular, sternoclavicular, temporomandibular, ankle, midtarsal and the metatarsophalangeal and PIP in the feet $[32,33]$. This is an extended joint count, as unlike in RA where the minimized 28 joint count performs well, in PsA, minimized joint count underestimates disease activity, since it does not assess arthritis in the lower extremities and in the DIP joints. Therefore, GRAPPA and OMERACT recommended the use of the 66/68 swollen and tender joint count. Despite the fact that joint assessment is a routine part of the daily clinical practice of rheumatologists, the accurate assessment of joint swelling may not be simple. In a reliability study that included rheumatologists who were experienced in assessing PsA and AS patients, the agreement on swollen joint count was only moderate (ICC 0.50) [34]. Lower agreement was achieved when dermatologists assessed PsA patients for joint swelling (ICC 0.30) [15]. Higher levels of agreement were achieved for assessment of joint tenderness (ICC 0.81 for rheumatologists and ICC 0.73 for dermatologist). Tender and swollen joint counts are sensitive to change, and can distinguish between treatment arm and placebo in clinical drug trials [35]. Despite the challenges in assessing joint swelling, joint count reflects the burden of peripheral inflammation, and it is part of any composite outcome measure used to determine the effectiveness of treatments in PsA. The widespread use of ultrasound as an adjunct for joint examination may improve the reliability of joint assessment in clinical practice in the future.

\section{Enthesitis}

Enthesitis is an inflammation at the attachment site of a tendon or ligament to the bone, and can involve both axial and peripheral joints. McGonagle et al. suggested that enthesitis lies in the basis of most, if not all, of the clinical manifestations of PsA [36]. Clinical assessment of enthesitis includes palpation and application of local pressure at the enthesis site. The presence of enthesitis is suggested by the detection of swelling, or more commonly tenderness, on palpation of the enthesis. However, studies that used ultrasound imaging as the gold standard for enthesitis found that clinical assessment underestimates the diagnosis of enthesitis [37, 38]. Enthesitis can affect any enthesis site in the body; however, most frequently it affects attachment sites in the lower extremities. Several enthesitis scores have been developed in an attempt to reflect the burden of enthesitis in an individual patient; each one includes different combination and number of entheseal sites.

The Mander enthesitis Index (MEI) was developed for assessment of enthesitis in ankylosing spondylitis (AS) patients [39]. It involves the assessment of 66 entheseal sites for the level of tenderness on palpation that is graded from 0 to 3 . The score has been criticized for being time-consuming and not practical for clinical use, due to the large number of entheseal sites examined. Furthermore, the large number of assessed sites and the grading of tenderness in each site can contribute to high inter-observer and intra-observer variability, thus reducing the reliability of the tool. Finally, the overlap of several of the included sites with fibromyalgia tender points complicates the assessment.

The Maastricht Ankylosing Spondylitis Enthesis Score (MASES) was derived from the 66 sites of the MEI after the recognition that the latter is not feasible for clinical use [40]. It is recommended by the Assessment of SpondyloArthritis international Society (ASAS) group for use in clinical trial of $\mathrm{SpA}$ and assesses the presence of tenderness in 13 entheseal sites, including bilateral first and 7th costochondral joints, 
posterior superior iliac spines, anterior superior iliac spines, iliac crests, Achilles tendons and 5th lumbar spinous process. This score was found to have only moderate reproducibility in patients with PsA and AS (ICC of 0.56) [34]. However, in several clinical drug trials in AS and PsA patients, it was able to discriminate between the treatment arm and the placebo and has shown responsiveness over time [27, 41•]. The Leeds Enthesitis Index (LEI) is the only enthesitis score that was developed to assess enthesitis specifically in PsA patients, in contrast to other scores that were developed for AS or SpA patients in general [42]. It records the presence of tenderness in six sites that were selected based on data reduction to reflect the most commonly involved sites in PsA. These sites included bilaterally: Achilles tendon insertion, medial femoral condyles and lateral epicondyles of the humerus. An additional enthesitis score is the Spondyloarthritis Research Consortium of Canada (SPARCC) that developed a tool for assessment of enthesitis in 16 entheseal sites in patients with SpA [43]. The following insertion sites are assessed bilaterally for tenderness: Achilles tendon, plantar fascia, patellar tendon to the base of the patella, quadriceps tendon to the patella, supraspinatus insertion to the greater tuberosity of the humerus and the medial and lateral epicondyles. The selection of the sites was based on the most commonly involved sites on imaging studies in patients with SpA. Shorter versions of SPARCC that include six and eight sites were found to be more reproducible than the longer version (ICC of 0.81) [34].

The performance of several of these tools, MEI, LEI, SPARCC and MASES, was compared in PsA patients after a change of disease modifying therapy over a period of 6 months. The LEI and SPARCC were found to be the most responsive over time. A minimal floor effect was observed for the LEI. All indices correlated strongly with other measures of disease activity (Table 1) [34].

\section{Dactylitis}

Dactylitis is a diffuse swelling of the whole finger or toe. The underlying lesion is a combination of inflammation in the joint and the adjacent tendon sheath. Dactylitis is one of the characteristic clinical features of PsA, and it helps to distinguish PsA from other types of arthritis such as RA. Dactylitis affects $16-48 \%$ of the patients with PsA, and is associated with future radiographic joint damage $[6,44 \cdot \bullet]$. The only validated tool that was developed to quantify psoriatic dactylitis is the Leeds Dactylitis Index [45]. In this method, the extent of swelling of each affected digit is quantified by measuring its circumference and comparing it to the contralateral digit. The ratio of the circumferences between the affected and unaffected contralateral digits is calculated. In addition, the degree of tenderness is also assessed by squeezing the affected digit, since chronic, non-tender dactylitic swelling that can occur in PsA, does not represent active inflammation. The total score is the sum of scores for each digit and represents the burden of dactylitis. The method was found to be reproducible (ICC of 0.70) in patients with PsA and AS in a reliability study that included rheumatologists who were experts in the field [34]. However, in several clinical trials, the assessment of dactylitis included either the number of digits with dactylitis or the assessment of tenderness and swelling on a $0-3$ score. Using this method, infliximab was proven effective for dactylitis [46].

\section{Spinal Assessment}

There is currently no agreed definition for axial involvement in patients with PsA. Axial involvement can present with inflammation of the sacroiliac joints (sacroiliitis) or the vertebral joints (spondylitis). It is the hallmark manifestation of AS; however, it tends to be less severe in patients with PsA. The occurrence of axial involvement in patients with PsA ranges from $15 \%$ to $43 \%$ [47-49]. Currently, there are no specific outcome measures for assessment of axial disease that were developed specifically for PsA. The Bath Ankylosis Spondylitis Disease Activity Index (BASDAI) is a six-item scale on which respondents rate the degree of back and joint pain and stiffness. The score ranges from 0 to 10 and is aimed to provide a global score for the entire spectrum of disease manifestations [50]. The validity of BASDAI was assessed in patients with PsA and was found to correlate well with patient perception of disease activity, but was unable to discriminate between high and low disease activity states as defined by treatment change [51]. The Ankylosing Spondylitis Disease Activity Score (ASDAS) is

Table 1 Comparison of sites assessed by the different enthesitis indices

\begin{tabular}{llll}
\hline & MASES & LEI & SPARCC \\
\hline $1^{\text {st }} \& 7^{\text {th }}$ costochondral joints & $\mathrm{X}$ & & \\
Posterior superior iliac spine & $\mathrm{X}$ & & \\
Anterior superior iliac spine & $\mathrm{X}$ & & \\
Iliac crest & $\mathrm{X}$ & & \\
Achilles tendon & $\mathrm{X}$ & $\mathrm{X}$ & $\mathrm{X}$ \\
$5^{\text {th }}$ lumbar spinous process & & & \\
Medial femoral condyle & & $\mathrm{X}$ & \\
Lateral epicondyle of humerus & & $\mathrm{X}$ & $\mathrm{X}$ \\
Plantar fascia & & $\mathrm{X}$ \\
Patellar tendon to patella & & $\mathrm{X}$ \\
Quadriceps tendon to patella & & & $\mathrm{X}$ \\
Supraspinatus to humerus & & $\mathrm{X}$ \\
Medial epicondyle of humerus & & $\mathrm{X}$ \\
\hline
\end{tabular}

MASES Maastricht Ankylosing Spondylitis Enthesis Score; LEI Leeds Enthesitis Index; SPARCC Spondyloarthritis Research Consortium of Canada 
a novel measurement tool to assess disease activity in patients with AS. It was developed because of the limitation of BASDAI being totally patient-derived. ASDAS is a calculated using a formula that incorporates three questions from the BASDAI questionnaire in addition to a patient global assessment score and an inflammatory marker (ESR or CRP) [52]. A study that compared the performance of BASDAI and ASDAS among patients with PsA who had axial involvement found that both scores had similar ability to discriminate between high and low disease activity states. Therefore, both tools can probably be used to measure axial activity in PsA patients [53].

Spondylitis can lead to limitation in range of motion of the spine as a result of ankylosis of the vertebrae. The damage can affect any part of the spine. Several measures have been developed to quantify the mobility and to objectively assess the severity of the damage to the spine in patients with AS. The Bath Ankylosing Spondylitis Metrology Index (BASMI) was developed to clinically assess spinal mobility [54]. It includes the following five items: cervical rotation as measured by the angle to which the patient can rotate the head to each side; cervical extension as measured by the distance between the targus of the ear to the wall (targus to wall distance); lumbar flexion as measured by the modified Schober test; lumbar side flexion as measured by the difference between the distance from the third finger and the floor when the patient stands straight and when the patient bends to the side; and intermalleolar distance as measured by the distance between the two malleoli when the patient lies down with the hips fully abducted. Each item is scored from 0 to 10 based on individually defined cutoff points. The BASMI has been used in clinical trials in AS patients. BASMI has been borrowed for use in PsA patients since no such index has been specifically developed for that group. A study that involved rheumatologists with expertise in the field of $\mathrm{SpA}$ compared the reliability of several metrology indices among patients with AS and PsA. The study has found that these metrology measures can be reliably used among patients with PsA [55].

\section{Function and Quality of Life}

The most commonly used measures of function and quality of life in PsA are the Health Assessment Questionnaire (HAQ), the Medical Outcome Study Short Form Health Survey (SF-36) and the Disease Life Quality Index (DLQI).

The HAQ was originally developed for the assessment of disability in patients with RA. It includes 20 questions about pain and the ability to perform activities of daily living [56]. The derived score ranges from 0 to 3 , with higher numbers reflecting greater disability. A modification of the HAQ for patients with spondyloarthritis (HAQ-S) includes questions about functions that are more likely to be affected in patients with spinal involvement [57]. The HAQ has been validated for patients with PsA and has been shown to be related to disease activity [58]. Both, HAQ and HAQ-S correlated similarly with clinical measures of function and pain in patients with PsA, suggesting that the modified version of the HAQ does not add significant information to that provided by the regular HAQ [58].

The SF-36 is a generic quality of life questionnaire consisted of eight sections: physical functioning, pain, vitality, social functioning, mental health, general health perception, role limitations due to physical problems and role limitations due to personal and emotional problems [59]. The score can be collapsed into two components: the physical component score (PCS) and the metal component score (MCS). The lower scores in SF-36 reflect decreased quality of life. The SF-36 has been validated in PsA patients and was found to correlate with measures of function and pain and could also discriminate between patients and healthy individuals [60].

The DLQI was developed to measure quality of life in patients with various skin conditions. It includes ten questions about the impact of the skin disease on work, social activities and personal relationship [61]. The score has been validated for the assessment of psoriasis and showed discrimination and responsiveness [62]. The DLQI is commonly used in clinical trial in patients with psoriasis and PsA for the assessment of the effect of the skin disease.

\section{Composite Outcome}

The global assessment of PsA is complicated by the heterogenous nature of the disease that can manifest in various

Table 2 Comparison of items included in composite measures for PsA

\begin{tabular}{lllll}
\hline & DAPSA & CPDAI & PASDAS & PsAJAI \\
\hline Patient global assessment & $\mathrm{X}$ & & $\mathrm{X}$ & $\mathrm{X}$ \\
Patient assessment of pain & $\mathrm{X}$ & & & $\mathrm{X}$ \\
Physician global assessment & & & $\mathrm{X}$ & $\mathrm{X}$ \\
Joint assessment & $\mathrm{X}$ & $\mathrm{X}$ & $\mathrm{X}$ & $\mathrm{X}$ \\
Inflammatory markers* & $\mathrm{X}$ & & $\mathrm{X}$ & $\mathrm{X}$ \\
Assessment of psoriasis & & $\mathrm{X}$ & & \\
Assessment of dactylitis & & $\mathrm{X}$ & $\mathrm{X}$ & \\
Assessment of enthesitis & & $\mathrm{X}$ & $\mathrm{X}$ & \\
Assessment of the spine & & $\mathrm{X}$ & & \\
Health-related quality of life** & & $\mathrm{X}$ & $\mathrm{X}$ & $\mathrm{X}$ \\
\hline
\end{tabular}

*measured by CRP or ESR

**measured by HAQ or SF-36

DAPSA Disease Activity index for Psoriatic Arthritis; CPDAI Composite Psoriatic Arthritis Disease Activity Index; PASDAS Psoriatic Arthritis Disease Activity Score; PSAJAI Psoriatic Arthritis Joint Activity Index 
clinical presentations. Furthermore, it has been argued that the psoriasis activity in the skin and nails should also be included in the global assessment of patients with psoriatic disease. A composite outcome measure is a way to incorporate the assessment of these various manifestations into a single measure that represents a global disease activity score. In the last few years, several composite outcome scores have been developed for assessing the activity of the various aspects of psoriatic disease. The Disease Activity index for Psoriatic Arthritis (DAPSA) assesses only the musculoskeletal component of the disease and includes three domains: a patient self-reported disease activity domain that is represented by patient global assessment of pain and disease activity using visual analogue scale; a joint assessment domain that is represented by tender and swollen joint counts,; and a acute phase reactants domain that is represented by ESR and CRP [63॰]. The variables that were found to reflect disease activity in the best way were selected following a principal component analysis. A different approach was taken for the development of the Composite Psoriatic Arthritis Disease Activity Index (CPDAI), which is based on five disease activity domains proposed by GRAPPA, including: joint disease, skin disease, enthesitis, dactylitis and spinal disease. Disease activity in each domain is graded from 0 to 3 giving a total score that ranges from 0 to 15 [64•]. The two tools were compared using data from a clinical trial of etanercept in PsA patients. Both of them were able to distinguish between the drug and placebo and were sensitive to change. However, only CPDAI distinguished between the two doses of etanercept, probably because it includes a domain for skin assessment [65••].

Psoriatic Arthritis Joint Activity Index (PsAJAI) is another composite outcome that was designed to measure response of psoriatic arthritis to treatment. [66]. Other response measures commonly used in clinical trials in PsA patients, such as the ACR response criteria, were borrowed from RA and therefore tend to underestimate disease activity in patient with PsA, as they do not assess joint sites that are commonly affected in these patients. The PsAJAI was developed from data from clinical trials of TNF $\alpha$ blockers in PsA using statistical modeling. The PsAJAI is a weighted sum of $30 \%$ improvement in the following six items: tender joint count, CRP, physician global assessment, pain, patient global assessment and HAQ. PsAJAI was found to perform better than two other response measures that are used in clinical trials in PsA, including the ACR20 and PsARC [66, 67••].

The GRACE study that was initiated by GRAPPA was aimed at developing a candidate composite disease activity index for PsA. The study was an international multicenter study that included 503 PsA patients who were followed for a 12-month period. The gold standard for defining an active disease state was based on the decision to change treatment. New indices were developed using regression analysis, Psoriatic Arthritis Disease
Activity Score (PASDAS), and empirically, based on physiciandefined cutoff for disease activity (arithmetic mean of desirability functions, AMDF). These new composite outcome measures were compared with existing composite measures; among them are the CPDAI and DAPSA (Table 2). Although all measures performed well, PASDAS was better able to distinguish between high and low disease activity states [68•0]. Further testing and comparison with existing tools is required to determine the properties of these new methods.

\section{Conclusion}

Psoriatic arthritis can manifest in various ways, which complicates its assessment. The availability of novel effective therapies required the development of new outcome measurement tools for assessment of the different aspects of the disease in clinical trials and observational studies. A significant progress has been made in our ability to quantify the activity of the different presentations of the disease. However, controversies still exists with respect to the optimal composite outcome measure for the assessment of disease activity in PsA patient. Efforts are underway by GRAPPA to better characterize the performance of the different measurement tools for this disease.

\section{Compliance with Ethics Guidelines}

Conflict of Interest L Eder declares no conflicts of interest. DD Gladman declares no conflicts of interest.

Human and Animal Rights and Informed Consent This article does not contain any studies with human or animal subjects performed by any of the authors.

\section{References}

Papers of particular interest, published recently, have been highlighted as:

- Of importance

-. Of major importance

1. Gelfand JM, Weinstein R, Porter SB, et al. Prevalence and treatment of psoriasis in the United Kingdom: a population-based study. Arch Dermatol. 2005;141:1537-41.

2. Brandrup F, Green A. The prevalence of psoriasis in Denmark. Acta Derm Venereol. 1981;61:344-6.

3. Gladman DD, Antoni C, Mease P, et al. Psoriatic arthritis: epidemiology, clinical features, course, and outcome. Ann Rheum Dis. 2005;64 Suppl 2:ii14-7.

4. Ibrahim G, Waxman R, Helliwell PS. The prevalence of psoriatic arthritis in people with psoriasis. Arthritis Rheum. 2009;61:1373-8.

5. Eder L, Gladman DD. Psoriatic arthritis: phenotypic variance and nosology. Curr Rheumatol Rep. 2013;15:316. Review of recent advances in classification of PsA and its various sub-types. 
6. Brockbank JE, Stein M, Schentag CT, et al. Dactylitis in psoriatic arthritis: a marker for disease severity? Ann Rheum Dis. 2005;64:188-90.

7. Sen D, Brasington R. Tight disease control in early RA. Rheum Dis Clin North Am. 2012;38:327-43.

8. Gladman DD, Mease PJ, Strand V, et al. Consensus on a core set of domains for psoriatic arthritis. J Rheumatol. 2007;34:1167-70.

9. Elkayam O, Ophir J, Yaron M, et al. Psoriatic arthritis: interrelationships between skin and joint manifestations related to onset, course and distribution. Clin Rheumatol. 2000;19:301-5.

10. Griffiths CE, Barker JN. Pathogenesis and clinical features of psoriasis. Lancet. 2007;370:263-71.

11. Fredriksson T, Pettersson U. Severe psoriasis - oral therapy with a new retinoid. Dermatologica. 1978;157:238-44.

12. Berth-Jones J, Grotzinger K, Rainville C, et al. A study examining inter- and intrarater reliability of three scales for measuring severity of psoriasis: Psoriasis Area and Severity Index, Physician's Global Assessment and Lattice System Physician's Global Assessment. Br J Dermatol. 2006;155:707-13.

13. Langley RG, Ellis CN. Evaluating psoriasis with Psoriasis Area and Severity Index, Psoriasis Global Assessment, and Lattice System Physician's Global Assessment. J Am Acad Dermatol. 2004;51:563-9.

14. Ashcroft DM, Wan Po AL, Williams HC, et al. Clinical measures of disease severity and outcome in psoriasis: a critical appraisal of their quality. Br J Dermatol. 1999;141:185-91.

15. Chandran V, Gottlieb A, Cook RJ, et al. International multicenter psoriasis and psoriatic arthritis reliability trial for the assessment of skin, joints, nails, and dactylitis. Arthritis Rheum. 2009;61:1235-42.

16. Kirby B, Fortune DG, Bhushan M, et al. The Salford Psoriasis Index: an holistic measure of psoriasis severity. Br J Dermatol. 2000;142:728-32.

17. Feldman SR, Fleischer Jr AB, Reboussin DM, et al. The selfadministered psoriasis area and severity index is valid and reliable. J Invest Dermatol. 1996;106:183-6.

18. Fleischer Jr AB, Feldman SR, Dekle CL. The SAPASI is valid and responsive to psoriasis disease severity changes in a multi-center clinical trial. J Dermatol. 1999;26:210-5.

19. - Puzenat E, Bronsard V, Prey S, et al. What are the best outcome measures for assessing plaque psoriasis severity? A systematic review of the literature. J Eur Acad Dermatol Venereol. 2010;24 Suppl 2:10-6. A systematic review that compared the performance of several clinical tools for assessment of psoriasis severity and concluded that PASI was the most valid and reproducible clinical severity score.

20. Ramsay B, Lawrence CM. Measurement of involved surface area in patients with psoriasis. Br J Dermatol. 1991;124:565-70.

21. Long CC, Finlay AY, Averill RW. The rule of hand: 4 hand areas $=2$ FTU $=1$ g. Arch Dermatol. 1992;128:1129-30.

22. Tiling-Grosse S, Rees J. Assessment of area of involvement in skin disease: a study using schematic figure outlines. $\mathrm{Br} \mathrm{J}$ Dermatol. 1993;128:69-74.

23. Langley RG, Krueger GG, Griffiths CE. Psoriasis: epidemiology, clinical features, and quality of life. Ann Rheum Dis. 2005;64 Suppl 2:ii18-23. discussion ii24-15.

24. Gladman DD, Anhorn KA, Schachter RK, et al. HLA antigens in psoriatic arthritis. J Rheumatol. 1986;13:586-92.

25. Rich P, Scher RK. Nail Psoriasis Severity Index: a useful tool for evaluation of nail psoriasis. J Am Acad Dermatol. 2003;49:206-12.

26. Aktan S, Ilknur T, Akin C, et al. Interobserver reliability of the Nail Psoriasis Severity Index. Clin Exp Dermatol. 2007;32:141-4.

27. Kavanaugh A, McInnes I, Mease P, et al. Golimumab, a new human tumor necrosis factor alpha antibody, administered every four weeks as a subcutaneous injection in psoriatic arthritis: Twentyfour-week efficacy and safety results of a randomized, placebocontrolled study. Arthritis Rheum. 2009;60:976-86.

28. Cassell SE, Bieber JD, Rich P, et al. The modified Nail Psoriasis Severity Index: validation of an instrument to assess psoriatic nail involvement in patients with psoriatic arthritis. J Rheumatol. 2007;34:123-9.
29. Gladman DD, Shuckett R, Russell ML, et al. Psoriatic arthritis (PSA)-an analysis of 220 patients. Q J Med. 1987;62:127-41.

30. Helliwell PS, Porter G, Taylor WJ. Polyarticular psoriatic arthritis is more like oligoarticular psoriatic arthritis, than rheumatoid arthritis. Ann Rheum Dis. 2007;66:113-7.

31. Deandrade JR, Casagrande PA. A seven-day variability study of 499 patients with peripheral rheumatoid arthritis. Arthritis Rheum. 1965;8:302-34.

32. Mease PJ, Antoni CE, Gladman DD, et al. Psoriatic arthritis assessment tools in clinical trials. Ann Rheum Dis. 2005;64 Suppl 2:ii49-54.

33. Gladman DD, Helliwell P, Mease PJ, et al. Assessment of patients with psoriatic arthritis: a review of currently available measures. Arthritis Rheum. 2004;50:24-35.

34. Gladman DD, Inman RD, Cook RJ, et al. International spondyloarthritis interobserver reliability exercise-the INSPIRE study: II. Assessment of peripheral joints, enthesitis, and dactylitis. J Rheumatol. 2007;34:1740-5.

35. Fransen J, Antoni C, Mease PJ, et al. Performance of response criteria for assessing peripheral arthritis in patients with psoriatic arthritis: analysis of data from randomised controlled trials of two tumour necrosis factor inhibitors. Ann Rheum Dis. 2006;65:1373-8.

36. McGonagle D, Khan MA, Marzo-Ortega H, et al. Enthesitis in spondyloarthropathy. Curr Opin Rheumatol. 1999;11:244-50.

37. Aydin SZ, Karadag O, Filippucci E, et al. Monitoring Achilles enthesitis in ankylosing spondylitis during TNF-alpha antagonist therapy: an ultrasound study. Rheumatology. 2010;49:578-82.

38. Borman P, Koparal S, Babaoglu S, et al. Ultrasound detection of entheseal insertions in the foot of patients with spondyloarthropathy. Clin Rheumatol. 2006;25:373-7.

39. Mander M, Simpson JM, McLellan A, et al. Studies with an enthesis index as a method of clinical assessment in ankylosing spondylitis. Ann Rheum Dis. 1987;46:197-202.

40. Heuft-Dorenbosch L, Spoorenberg A, van Tubergen A, et al. Assessment of enthesitis in ankylosing spondylitis. Ann Rheum Dis. 2003;62:127-32.

41. - Rudwaleit M, Claudepierre P, Kron M, et al. Effectiveness of adalimumab in treating patients with ankylosing spondylitis associated with enthesitis and peripheral arthritis. Arthritis Res Ther. 2010;12:R43. A clinical drug trial that used MASES to assess the effect of adalimumab vs. placebo on enthesitis in patients with AS. It found that MASES was able to discriminate between the treatment arm and the placebo and has shown responsiveness over time.

42. Healy PJ, Helliwell PS. Measuring clinical enthesitis in psoriatic arthritis: assessment of existing measures and development of an instrument specific to psoriatic arthritis. Arthritis Rheum. 2008;59:686-91.

43. Maksymowych WP, Mallon C, Morrow S, et al. Development and validation of the Spondyloarthritis Research Consortium of Canada (SPARCC) Enthesitis Index. Ann Rheum Dis. 2009;68:948-53.

44. •- Coates LC, Helliwell PS. Disease measurement - enthesitis, skin, nails, spine and dactylitis. Best Pract Res Clin Rheumatol. 2010;24:659-70. Review of outcome measures for assessment of the different manifestations of psoriatic disease.

45. Helliwell PS, Firth J, Ibrahim GH, et al. Development of an assessment tool for dactylitis in patients with psoriatic arthritis. J Rheumatol. 2005;32:1745-50.

46. Antoni C, Krueger GG, de Vlam K, et al. Infliximab improves signs and symptoms of psoriatic arthritis: results of the IMPACT 2 trial. Ann Rheum Dis. 2005;64:1150-7.

47. Gladman DD, Brubacher B, Buskila D, et al. Differences in the expression of spondyloarthropathy: a comparison between ankylosing spondylitis and psoriatic arthritis. Clin Invest Med. 1993;16:1-7.

48. Chandran V, Barrett J, Schentag CT, et al. Axial psoriatic arthritis: update on a longterm prospective study. J Rheumatol. 2009;36:2744-50.

49. Veale D, Rogers S, Fitzgerald O. Classification of clinical subsets in psoriatic arthritis. Br J Rheumatol. 1994;33:133-8.

50. Garrett S, Jenkinson T, Kennedy LG, et al. A new approach to defining disease status in ankylosing spondylitis: the Bath Ankylosing Spondylitis Disease Activity Index. J Rheumatol. 1994;21:2286-91. 
51. Taylor WJ, Harrison AA. Could the Bath Ankylosing Spondylitis Disease Activity Index (BASDAI) be a valid measure of disease activity in patients with psoriatic arthritis? Arthritis Rheum. 2004;51:311-5.

52. Lukas C, Landewe R, Sieper J, et al. Development of an ASASendorsed disease activity score (ASDAS) in patients with ankylosing spondylitis. Ann Rheum Dis. 2009;68:18-24.

53. Eder L, Chandran V, Shen H, et al. Is ASDAS better than BASDAI as a measure of disease activity in axial psoriatic arthritis? Ann Rheum Dis. 2010;69:2160-4.

54. Jenkinson TR, Mallorie PA, Whitelock HC, et al. Defining spinal mobility in ankylosing spondylitis (AS). The Bath AS Metrology Index. J Rheumatol. 1994;21:1694-8.

55. Gladman DD, Inman RD, Cook RJ, et al. International spondyloarthritis interobserver reliability exercise-the INSPIRE study: I. Assessment of spinal measures. J Rheumatol. 2007;34:1733-9.

56. Fries JF, Spitz P, Kraines RG, et al. Measurement of patient outcome in arthritis. Arthritis Rheum. 1980;23:137-45.

57. Daltroy LH, Larson MG, Roberts NW, et al. A modification of the Health Assessment Questionnaire for the spondyloarthropathies. J Rheumatol. 1990;17:946-50.

58. Blackmore MG, Gladman DD, Husted J, et al. Measuring health status in psoriatic arthritis: the Health Assessment Questionnaire and its modification. J Rheumatol. 1995;22:886-93.

59. Ware Jr JE, Sherbourne CD. The MOS 36-item short-form health survey (SF-36). I. Conceptual framework and item selection. Med Care. 1992;30:473-83.

60. Husted JA, Gladman DD, Farewell VT, et al. Validating the SF-36 health survey questionnaire in patients with psoriatic arthritis. J Rheumatol. 1997;24:511-7.

61. Finlay AY, Khan GK. Dermatology Life Quality Index (DLQI)-a simple practical measure for routine clinical use. Clin Exp Dermatol. 1994;19:210-6.

62. Mazzotti E, Barbaranelli C, Picardi A, et al. Psychometric properties of the Dermatology Life Quality Index (DLQI) in 900 Italian patients with psoriasis. Acta Derm Venereol. 2005;85:409-13.

63. - Schoels M, Aletaha D, Funovits J, et al. Application of the DAREA/DAPSA score for assessment of disease activity in psoriatic arthritis. Ann Rheum Dis. 2010;69:1441-7. A study that validated the DAPSA, a new composite outcome measure for assessment of disease activity, in PSA patients.

64. - Mumtaz A, Gallagher P, Kirby B, et al. Development of a preliminary composite disease activity index in psoriatic arthritis. Ann Rheum Dis. 2011;70:272-7. A study that describes the development and validation of CPDAI as a new composite outcome for assessment of disease activity in PSA.

65. •• FitzGerald O, Helliwell P, Mease P, et al. Application of composite disease activity scores in psoriatic arthritis to the PRESTA data set. Ann Rheum Dis. 2012;71:358-62. A study that compared the performance of two novel composite outcome measures for PsA in a clinical drug trial. The study found that both measures were able to distinguish between the drug and placebo and were sensitive to change. However, only CPDAI distinguished between the two doses of the medication.

66. Gladman DD, Tom BD, Mease PJ, et al. Informing response criteria for psoriatic arthritis. I: discrimination models based on data from 3 anti-tumor necrosis factor randomized studies. J Rheumatol. 2010;37:1892-7.

67. • Gladman DD, Tom BD, Mease PJ, et al. Informing response criteria for psoriatic arthritis (PsA). II: Further considerations and a proposal - the PsA joint activity index. J Rheumatol. 2010;37:255965. A study that describes the development and validation process of PSAJAI, a novel response measurement for PsA.

68. • Helliwell PS, Fitzgerald O, Fransen J, et al. The development of candidate composite disease activity and responder indices for psoriatic arthritis (GRACE project). Ann Rheum Dis. 2012. A study that reports the results of the GRACE project. An international multicenter study aimed at developing a candidate composite disease activity index for PSA. New indices were developed using regression analysis, Psoriatic Arthritis Disease Activity Score (PASDAS), and empirically, based on physician-defined cut-off for disease activity (arithmetic mean of desirability functions, $A M D F)$. These new composite outcome measures were compared with existing composite measures; among them are the CPDAI and DAPSA. 